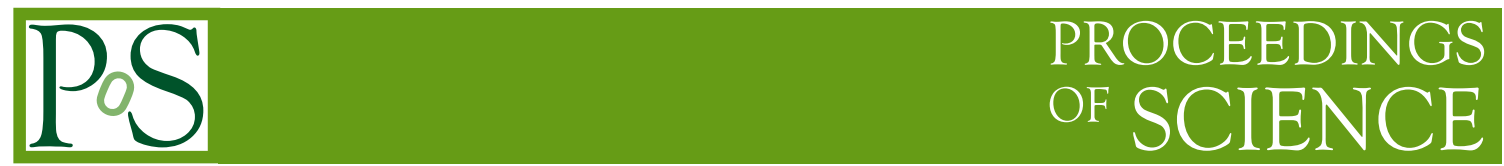

\title{
Searches for new physics with leptons in ATLAS
}

\author{
Xin Chen*† \\ Department of Physics, University of Wisconsin, Madison, W 53706, U.S.A. \\ E-mail: xi n. chen@ern. ch
}

Leptonic final states are rare and have relatively small backgrounds at a hadron collider. Many models of beyond the Standard Model physics predict new particles which may decay into high PT leptons and/or anomal ously large missing transverse energy. A number of exotic signatures, such as W', Z', SUSY, Leptoquarks, LRSM Majorana neutrinos and diboson resonances, are being searched for by ATLAS. The prospects and results of some of these searches with the early data coll lected by ATLAS in 2010, are presented here.

Wbrkshop on Discovery Physics at the LHC -Kruger 2010

December 05-10, 2010

Kruger National Park, Mpumalanga, South Africa

\footnotetext{
*Speaker.

†On behalf of theATLAS Collaboration.
} 

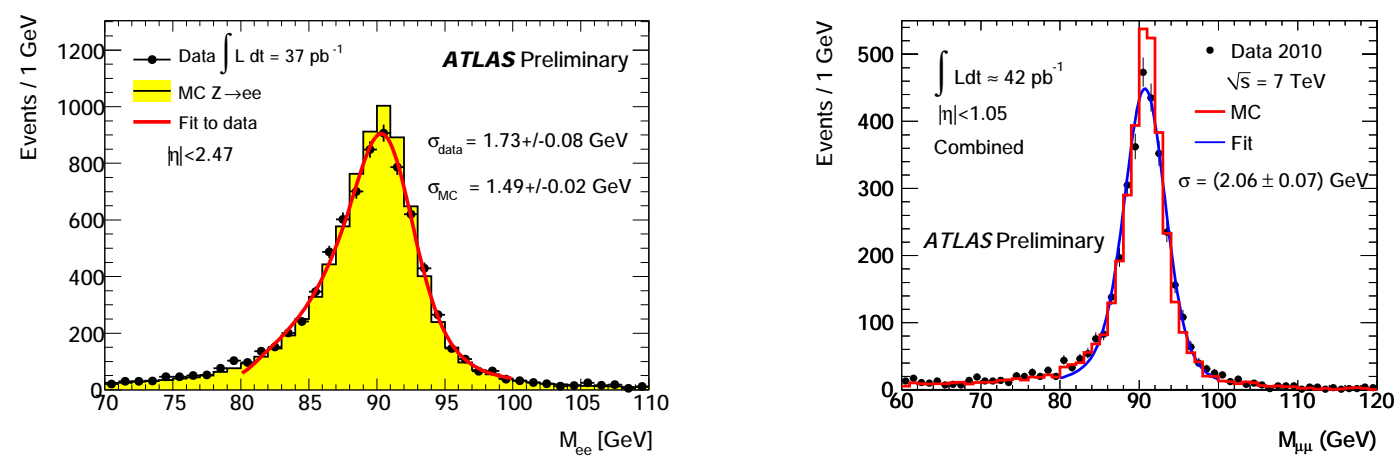

Figure 1: Left the $Z \rightarrow €$ invariant mass for dielectron pairs. Right: invariant mass distribution for oppositely charged and isol ated barrel dimuon pairs.

\section{Introduction}

Although the Standard Model (SM) has remarkable predictive powers for the known particle physics phenomena, some theoretical problems, such as the need for finetuning radiative corrections to the Higgs mass, have led physicists to search for new physics beyond the SM. In some of these models, new particles are predicted from extended gauge symmetries. These new particles can decay into high momentum leptons. Many models[1] predict the existence of new heavy gauge bosons: $W^{\prime}$ and $Z^{\prime}$. These are similar to the SM $W$ and $Z$ gauge bosons but with much heavier masses and undetermined coupling. In the Left-Right Symmetry model (LRSM), the $W^{\prime}$ may de cay into a right-handed Majorana neutrino, which then decays and leads to the possi ble observation of two same-sign leptons. Leptons can be al so found in the cascade decays of the supersymmetric (SUSY) particles such as gl uinos and sqarks, whose final state is characterized by leptons +jets + missing transverse energy $\left(\mathrm{Z}_{\mathrm{T}}\right)$. The observed symmetry between leptons and quarks has motivated the search for leptoquarks, hypothetical bosons carrying both lepton and quark quantum numbers, and decay into a lepton and a quark in the same generation. In the absence of a light Higgs, various models predict diboson resonances that can be produced via WW scattering, and which decay into one or more leptons.

ATLAS recorded about $45 \mathrm{pb}^{-1}$ of integrated luminosity of pp collisions at $\sqrt{\mathrm{s}}=7 \mathrm{TeV}$ in 2010. The detector has performed very well according to the design goals. Fig. 1 shows the dielectron and dimuon invariant mass distributions. Good agreement between data and MC in terms of $Z$ mass peak and resolution is observed. Leptons (electrons, muons and taus that decay leptonically) are efficiently triggered ${ }^{1}$. For $\mathrm{p}_{\mathrm{T}}>20 \mathrm{GeV}$, thefake lepton rejection factor is, roughly speaking, $10^{2}-10^{3}\left(10^{3}\right)$ for the electrons (muons). Analyses searching for new physics involving leptons with the very first data of ATLAS are presented here. For the ones with no available data results yet, prospects based on $\sqrt{\mathrm{s}}=7 \mathrm{TeV}$ or $14 \mathrm{TeV}$ Monte Carlo (MC) results are given.

\footnotetext{
${ }^{1}$ The L1 trigger efficiency is about 75-95\% (>99\%) for muons (electrons).
} 

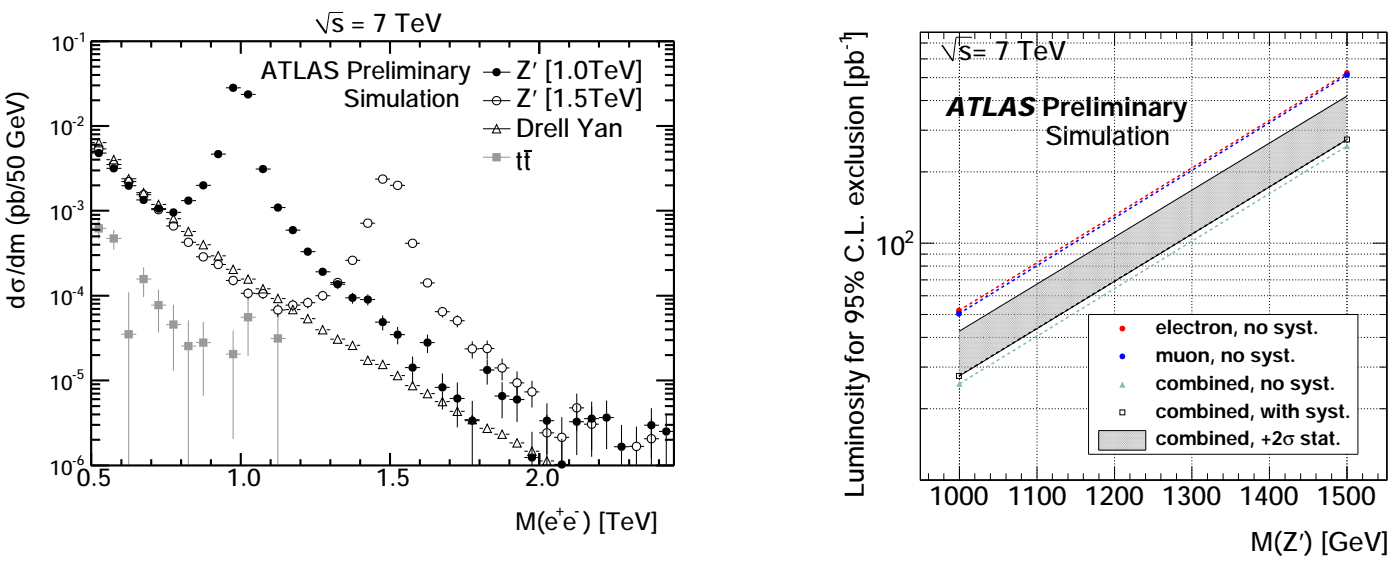

Figure 2: Right: dielectron invariant mass spectra after all cuts. Right: integrated luminosity expected to allow a $95 \%$ CL exclusion of theSSM $Z^{\prime}$ model, as a function of $Z^{\prime}$ mass.

\section{2. $Z^{\prime} \rightarrow \Subset \mu \mu$}

To search for a $Z^{\prime} \rightarrow$ eeor $Z^{\prime} \rightarrow \mu \mu$ signature, events with two good leptons with $\mathrm{p}_{\mathrm{T}}>20 \mathrm{GeV}$, $|\eta|<2.5$ and $\Sigma_{\Delta \mathrm{R}<0.3} \mathrm{p}_{\mathrm{T}}^{\mathrm{tracks}} / \mathrm{p}_{\mathrm{T}}^{\text {lepton }}<0.05$ are selected. The single lepton trigger efficiencies are about $98 \%$ for events that pass the final cuts. The dielectron invariant mass after all cuts and the exclusion sensitivity for the two channels are shown in Fig. 2, for $Z^{\prime}$ masses of 1.0 and $1.5 \mathrm{TeV}$ in the Sequential Standard Models(SSM) [2] with SM-likecouplings. TheZ' resolution in the dimuon channel is worse than in the dielectron channel, since the muon $\mathrm{p}_{\mathrm{T}}$ resolution deteriorates to about $10 \%$ (barrel) at $1 \mathrm{TeV}$, whereas the electron one stays around $1 \%$ owing to the $\mathrm{EM}$ calorimeter. Under the $Z^{\prime}$ peak, the main SM backgrounds are the Drell-Yan process $\left(Z / \gamma^{*} \rightarrow € e, \mu \mu\right)$ and $t t$. A $1.5 \mathrm{TeV} Z^{\prime}$ in SSM can be excluded with about $400 \mathrm{pb}^{-1}$ of data including systematics, while the current CDF limit is around $1 \mathrm{TeV}$ [3]. It is worth noting that $Z^{\prime}$ can al so be searched for in the $\tau \tau$ and $t \bar{t}$ decays mode if $Z^{\prime}$ prefers to couple to the third generation, but these channe s are more difficult to reconstruct and need higher luminosity to observe.

\section{3. $\mathrm{W}^{\prime} \rightarrow \mathrm{ev}, \mu v$}

To search for $\mathrm{W}^{\prime} \rightarrow \mathrm{ev}$ in data, ATLAS selects events with (1) at least one primary vertex with at least 3 associated tracks, (2) no jets with large calorimeter noise, (3) exactly one medium electron with $\mathrm{p}_{\mathrm{T}}>20 \mathrm{GeV}$ within the tracking acceptance and well isolated, (4) $\mathrm{E}_{\mathrm{T}}>25 \mathrm{GeV}$ to reject low $Z_{T}$ events such as QCD multijet. Fig. 3 shows the transverse mass $\left(m_{T}\right)$ distribution after all cuts and the $W^{\prime}$ exclusion limits by counting the events in the $m_{T}>0.7 \mathrm{~m}_{W^{\prime}}$ region. Very good agreement between the $m_{\pi}$ distributions in data and SM prediction is observed. The main backgrounds left after all cuts areW/Z and QCD multijet, which are small compared to the signal . A $465 \mathrm{GeV} \mathrm{W}^{\prime}$ in the SSM is excluded at $95 \% \mathrm{CL}$ with $0.317 \mathrm{pb}^{-1}$ of datain the ev channel al one, which is consistent with the limit of $1 \mathrm{TeV}$ from D0 [4]. A similar sensitivity is expected in the $\mathrm{W}^{\prime} \rightarrow \mu v$ channel. With about $500 \mathrm{pb}^{-1}$ of data, a $2 \mathrm{TeV} \mathrm{W}^{\prime}$ (SSM) can be excluded at $95 \% \mathrm{CL}$ with thee $v$ and $\mu \nu$ channels combined. 

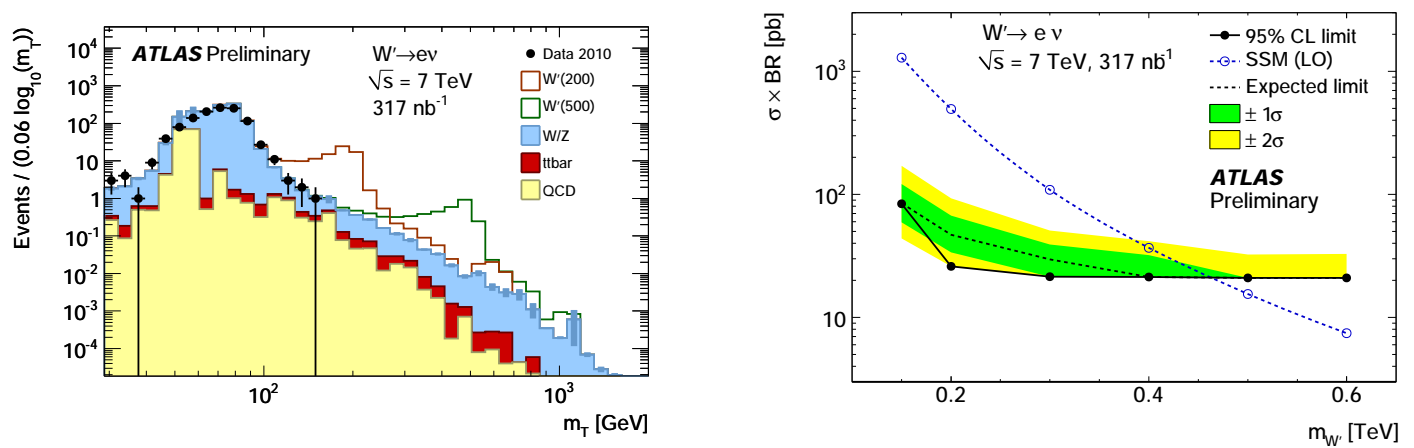

Figure 3: Left: $\mathrm{m}$ spectrum after the final cuts. Right: Limits on $\mathrm{W}^{\prime}$ production as a function of $\mathrm{W}^{\prime}$ mass with $317 \mathrm{nb}^{-1}$ of data.
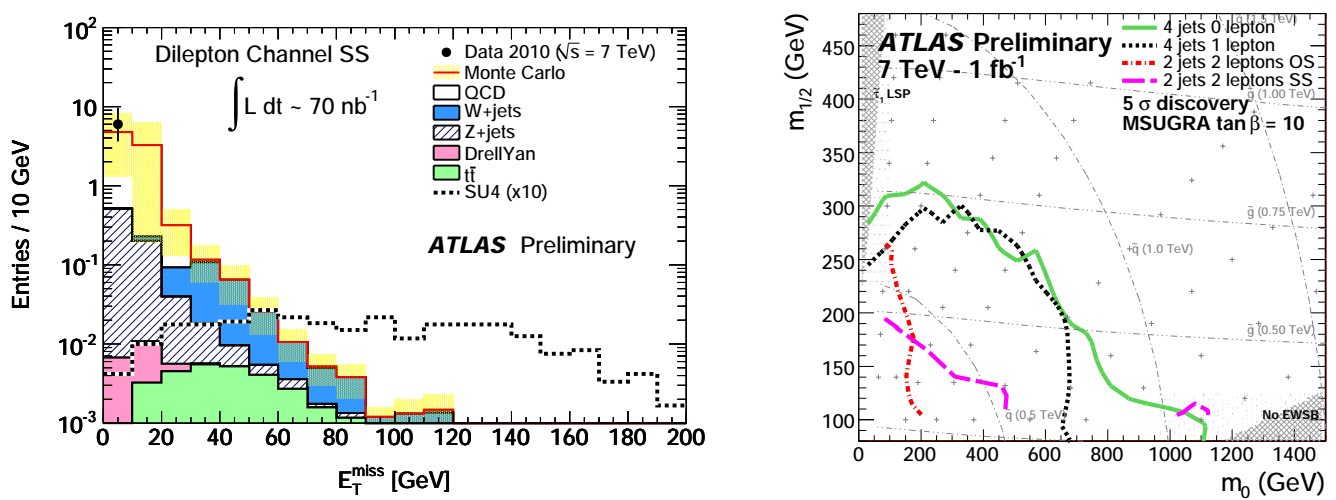

Figure 4: Left the as a function of $m_{0}$ and $m_{1 / 2}$ in the mSUGRA model.

\section{SUSY leptonic decay modes}

SUSY introduces a new symmetry between the bosons and fermions, protects the SM Higgs mass, al lows a unification of the el ectroweak and strong forces, and many models of SUSY provide a dark matter candidate. If SUSY exists, gluino/squark pairs can be copiously produced (subject to the mass scale) at LHC, and one or more leptons can be found in the cascade decays of the super particles, together with multiple jets and large $\mathrm{Z}_{\mathrm{T}}$. The $\mathrm{Z}_{\mathrm{T}}$ distribution in the same-sign 2lepton channel and the $5 \sigma$ discovery reach in the mSUGRA [5] model are shown in Fig. 4 . It is expected that with $1 \mathrm{fb}^{-1}$ of data, a large corner of parameter space in the mSUGRA model can be discovered, which corresponds to gluino and squark masses of up to about $700 \mathrm{GeV}$.

\section{Leptoquarks}

Leptoquark (LQ) can be pair-produced via QCD and LQ-lep-LQ couplings at LHC. The se lection requires at least 2 leptons and 2 jets, large $p_{T}$ sum of leptons and jets ( $\gtrsim 500 \mathrm{GeV}$ ), large 

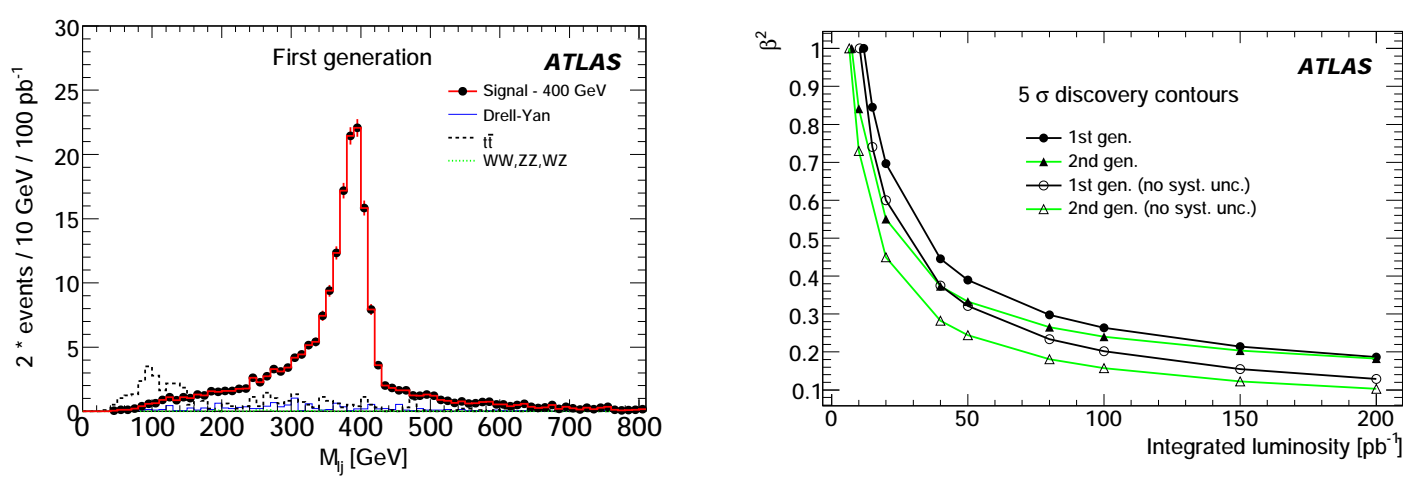

Figure 5: Left: electron-jet invariant mass for the 1st generation $\mathrm{LQ}$ with $\mathrm{m}_{\mathrm{Q}}=400 \mathrm{GeV}$ after all cuts and normalized to $100 \mathrm{pb}^{-1}$. Right: $5 \sigma$ discovery potential for $1 s t$ and 2 nd generation LQs with $\mathrm{m}_{\mathrm{Q}}=400$ $\mathrm{GeV}$.

dilepton mass ( $m_{\mathrm{I}} \gtrsim 120 \mathrm{GeV}$ ). Lepton-jet pair ambiguity is resolved by minimizing the mass difference of such two pairs. The dominant backgrounds after all cuts are Drell-Yan/Z and tt. For $\beta=\mathrm{BR}(\mathrm{LQ} \rightarrow \mathrm{Iq})=1$, D0 limit on the LQ mass is around $250 \mathrm{GeV}$ [6]-[7]. The electron-quark pair invariant mass and the ATLAS $5 \sigma$ discovery potential (at $\sqrt{\mathrm{s}}=14 \mathrm{TeV}$ ) are shown in Fig. 5.

\section{6. $W_{R} \rightarrow 2 I+2 j$ in LRSM}

In the LRSM, the $\mathrm{W}^{\prime}$ couples to right-handed fermions (thus denoted as $\mathrm{W}_{\mathrm{R}}$ ). It can decay into a lepton and a heavy neutrino $N_{R}$, which then decays into a lepton and two quarks. Due to the Majorana nature of $\mathrm{N}_{\mathrm{R}}$, these two leptons can have the same charge, which leads to the observation of lepton number violation ( $\Delta \mathrm{L}=2)$ with no $\mathrm{ET}_{\mathrm{T}}$. In the analysis, at least 2 leptons and 2 jets are required, with large pт sum of leptons and jets ( $>700 \mathrm{GeV}$ ) and large di lepton mass (>300 GeV). The lepton+2jets combination ambiguity for $N_{R}$ is resolved by taking the one with the smaller mass. The current D0 limit on $W_{R}$ is about $750 \mathrm{GeV}$ [8] in the decay mode of $W^{\prime} \rightarrow$ tb. Fig. 6 shows thee +2 jets invariant mass distribution and the $5 \sigma$ discovery contour for two signal samples with no flavor mixing (at $\sqrt{\mathrm{s}}=14 \mathrm{TeV}$ ): (1) $\mathrm{m}_{\mathrm{V}_{\mathrm{R}}}=1800 \mathrm{GeV}, \mathrm{m}_{\mathrm{N}_{\mathrm{R}}}=300 \mathrm{GeV}$; (2) $\mathrm{m}_{\mathrm{V}_{\mathrm{R}}}=1500$ $\mathrm{GeV}, \mathrm{m}_{\mathrm{N}_{\mathrm{R}}}=500 \mathrm{GeV}$.

\section{Diboson resonaces}

In the absence of a light Higgs with $\mathrm{m}_{\mathrm{H}} \lesssim 700 \mathrm{GeV}$, new physics must exist at some high en ergy scale based on the arguments of vector boson scattering unitarity [9], possibly in the form of diboson resonances. To search for them, di boson resonance signals are generated with an effective Chiral Lagrangian. Two forward tagging jets plus central jet veto are required for theanalysis. The main backgrounds areW $/ Z+4 j$ ets and di boson+2jets. Signal s can be reconstructed in the subchannels of WW/WZ $\rightarrow|v \mathrm{j} \mathrm{j}, \mathrm{WZ} \rightarrow \mathrm{j} \mathrm{jll} / \mathrm{I} v| \mathrm{l}$ and $\mathrm{ZZ} \rightarrow v v \mid \mathrm{I}$. However, to establish a discovery of these subchannels, about $55-235 \mathrm{fb}^{-1}$ of data (at $\sqrt{\mathrm{s}}=14 \mathrm{TeV}$ ) is needed. 

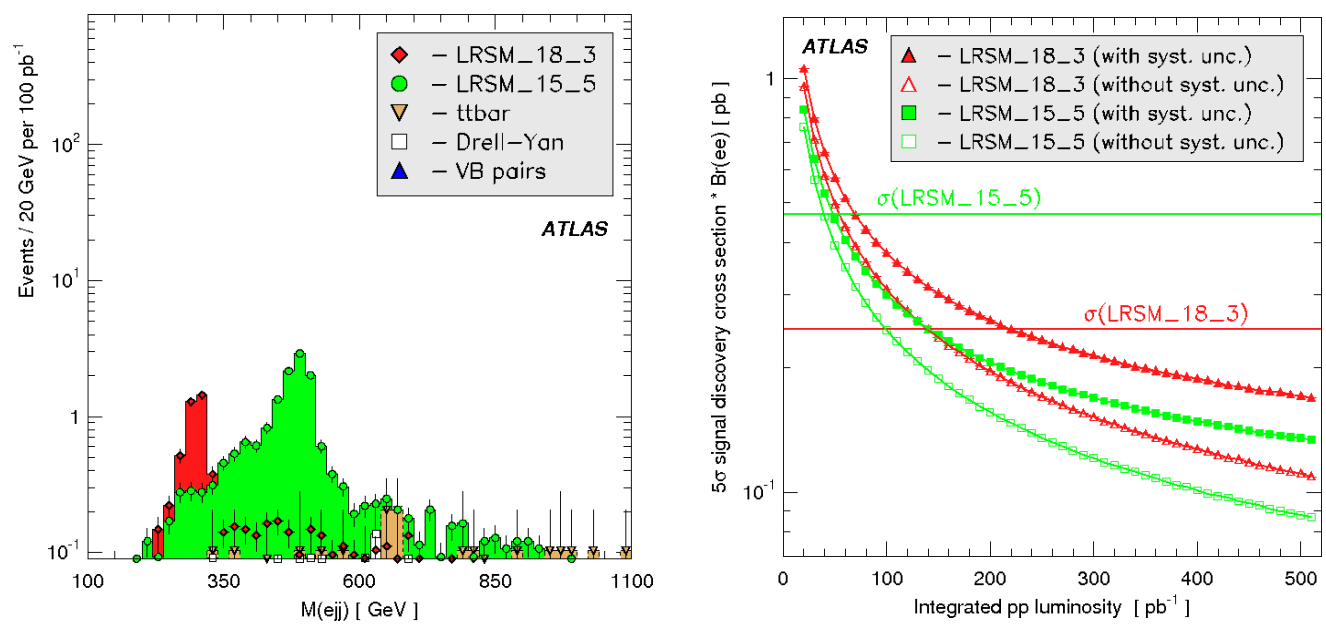

Figure 6: Left: the ej $\mathrm{j}$ invariant mass for $\mathrm{N}_{\mathrm{R}}$ candidates after the background suppression cuts in the dielectron channel (normalized to $100 \mathrm{pb}^{-1}$ ). Right: the necessary signal $\sigma \times \mathrm{BR}$ for a $5 \sigma$ discovery as a function of integrated luminosity in the dielectron channel.

\section{Conclusions and outlooks}

Many new physics models include leptons in the final state, which can be efficiently triggered and cleanly reconstructed by ATLAS. The early data of SM W/Z benchmarks is available to calibrate the leptons and check against the MC prediction and detector simulation. In general, the ATLAS MC is well tuned and agrees with data. A W' with SM coupling and mass of $465 \mathrm{GeV}$ is excluded at $95 \% \mathrm{CL}$ by $0.317 \mathrm{pb}^{-1}$ of initial ATLAS data collected in 2010. Distributions of SUSY-sensitive variables show no evidence of SUSY based on $70 \mathrm{nb}^{-1}$ of data. Prospects and expected sensitivities of searches for $W^{\prime}, Z^{\prime}$, SUSY, Leptoquark, $W_{R}$ and Majorana neutrinos in LRSM, and diboson resonances are al so summarized in this note. Data results based on higher luminosities have yet to come, but the sensitivities will surpass Tevatron in many new physics search channels as new data continues to be collected in year 2011 and onwards.

\section{References}

[1] C. Amsler et al., Reviews on W' and Z', Phys. Lett B 667 (2008) 1.

[2] G. Altarelli, B. Mele, M.Ruiz-Altaba, Z. Phys. C 45 (1989) 109.

[3] T. Aaltonen et al., Phys. Rev. Lett 102 (2009) 091805.

[4] V. M. Abazov et al., Phys. Rev. Lett 100 (2008) 031804.

[5] A.H. Chamseddine, R. Arnowitt, Pran Nath, Phys. Rev. Lett 49 (1982) 970.

[6] V. M. Abazov et al., Phys. Lett B 636 (2006) 183.

[7] V. M. Abazov et al., Phys. Rev. D 71 (2005) 071104.

[8] V. M. A bazov et al., Phys. Rev. Lett 100 (2008) 211803.

[9] B.W. Le, C. Quigg, H.B. Thacker, Phys. Rev. Lett. 38 (1977) 883. 